\title{
Evaluation of bearing capacity of a beam on an elastic foundation using the methods of the theory of limiting balance
}

\author{
Vasil Nizameev*1[0000-0001-8525-7611], Fanzil Basharov ${ }^{1}$, and Luiza Nizameeva ${ }^{1}$ \\ ${ }^{1}$ Kazan State University of Architecture and Engineering, 420043 Kazan, Russia
}

\begin{abstract}
The analytical expressions and graphs for determining a limiting load have been obtained using the methods of the theory of limiting balance. The processes of strain of the beams after formation of a plastic hinge until their complete destruction have been investigated. The dependences of a bearing capacity of the beam on its parameters and characteristics of the foundation soils have been obtained. The regularities of formation of plastic hinges have been revealed depending on a place of application of the load. The limiting values of the load have been calculated analytically, and the forms of deformation of the beam have been established at the time of the appearance of the first plastic strains and in the limiting state. The comparative analysis of the research results of the adopted model with the numerical studies of the elastoplastic beam has been carried out.

Keywords. Beam on an elastic foundation, the theory of limiting balance, limiting moment.
\end{abstract}

\section{Introduction}

The object of research is a beam on a continuous elastic foundation loaded with a parametric concentrated force. The purpose of the study is to evaluate the bearing capacity of a beam on an elastic foundation using the methods of the theory of limiting equilibrium. The modern practice of calculating the structures on a foundation being strained mainly uses the finite element method implemented in various software packages. The disadvantage of these theories and methods of calculations is that elastic models are used for beams or slabs having different models of the foundation, which does not allow determining a true margin of safety, since a limiting state of the structure is accompanied by the appearance of plastic strains in it. Therefore, the methods of limiting balance make it possible to supplement the existing gap in this issue. The research topic is relevant because structural elements are often found lying on an elastic foundation in construction practice, such as strip foundations of buildings, railway sleepers, etc. Calculation of a beam on an elastic foundation in general is reduced to solving a contact problem between the beam and the foundation. The usual solution of such contact problem has great difficulties and is not suitable for engineering calculations. Therefore, to solve engineering problems associated with the calculation of a beam on an elastic foundation, approximate approaches are used. At the same time, a dependence is

"Corresponding author: nizameev_kisi@mail.ru 
established between the reactive resistance and the settlement of the elastic foundation, for example, according to the hypothesis of a proportional dependence between the reaction and the settlement (the hypothesis of the Winkler foundation).

A promising approach to solving this problem is the use of the finite element method and the limit equilibrium method.

\section{Materials and methods}

The methods of the theory of limiting balance, first proposed in the work [1], are now widely used in assessing the bearing capacity of various building structures. Many publications are devoted to development and improvement of the methods of the theory of limiting balance [2-9], especially regarding calculation of the reinforced concrete structures [3-4, 10-12], including the beam ones [11-12]. At the same time, these methods are practically not used when calculating the beams and slabs lying on a foundation being strained. The works [9-18] are rare exceptions.

The theory of calculating the structures, lying on an elastic foundation, has been widely developed in the works [9-20], where the following models of the elastic foundation are used: a Winkler's model (otherwise called a model of the coefficient of subgrade reaction); a model of the elastic homogeneous isotropic half-space; a model of the elastic homogeneous isotropic layer; a model of the foundation with two elastic characteristics. In some works, other models are used too, which are mainly combinations of the above. The modern practice of calculating the structures on a foundation being strained mainly uses the finite element method implemented in various software packages. The disadvantage of these theories and methods of calculations is that elastic models are used for beams or slabs having different models of the foundation, which does not allow determining a true margin of safety, since a limiting state of the structure is accompanied by the appearance of plastic strains in it. Therefore, the methods of limiting balance, which in this work are implemented on the example of a beam under a concentrated force, make it possible to supplement the existing gap in this issue.

\section{Results and discussion}

\subsection{Limiting load corresponding to the appearance of plastic strains}

A beam lying on an elastic Winkler's foundation is considered. A rigid-plastic model of strain is adopted for the beam. A bearing capacity of the beam at this stage of the calculation will be associated with the achievement of a bending moment of the limiting value (a yield point) in any section of the beam. In this case, the influence of the lateral force on the bearing capacity will be considered insignificant and not taken into account.

The strains for the accepted model of the beam in any section will be equal to zero as long as a bending moment in the section is less than a yield moment: $M<M_{y}$, and the strains appear at $M=M_{T}$. For a multi-strength beam, for example, a reinforced concrete beam with different reinforcement of the upper and lower layers, the condition for the absence of strains will be written as: $-M_{y}^{-}<M<M_{y}$, where $M_{y}$ is a limiting moment for the case of positive bending (the lower fibers of the beam are stretched), $M_{y}^{-}$is a limiting moment for the case of negative bending (the upper fibers are stretched).

\subsection{A yield point for the force, applied in the middle}

First, let us consider the simplest case of loading the beam with a concentrated force applied in the middle. The task is to determine the limiting value of the concentrated force (a yield 
point $-\mathrm{P}_{\mathrm{T}}$ ), corresponding to the appearance of plastic strains. The solution is sought using the kinematic and static methods the theory of limiting balance, which, respectively, give an approximation from above and from below to a true value of the limiting load. In this case, let us assume that bottom of the beam does not come off the foundation due to a sufficient swamp weight - a constant load evenly distributed along the entire length of the beam.

With an increase in the parametric load $P$, the beam, before the appearance of plastic strains, is immersed in the elastic foundation as an absolutely rigid body, overcoming the bearing reaction $r=k b v=P / l$, where $k$ is a coefficient of foundation subgrade reaction, $b$ is a width of the beam foundation, $v-$ a foundation settlement.

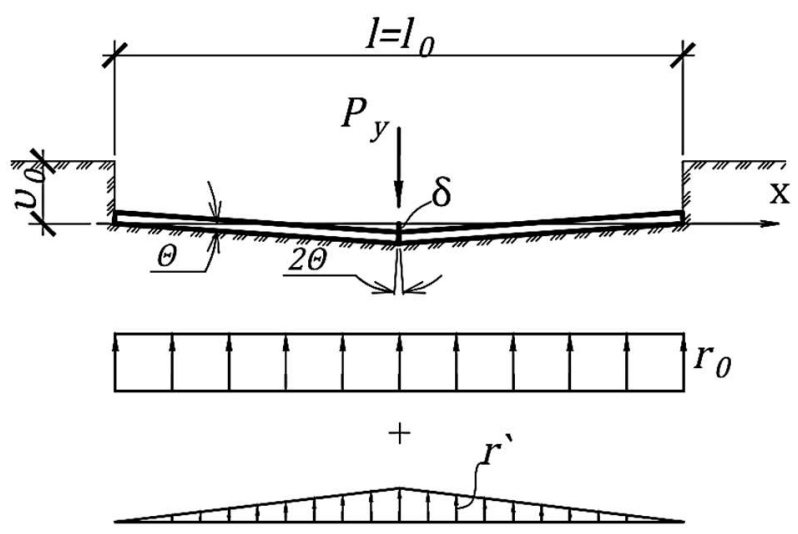

Fig. 1. Schemes of the beam strains at the moment of forming a plastic hinge.

Since the greatest bending moment occurs under the force $P$, it is obvious that the first plastic strains will occur in the middle of the beam in the form of formation of a plastic hinge (see diagram in Figure 1), the opening of which is determined by the angle $2 \theta=4 / l$, where is an infinitesimal displacement of the point of application of the force $P$. The reaction of the foundation in this case consists of two parts:

$r(x)=r_{0}+r^{\prime}(x)$, where $r_{0}=k b v_{0}=P_{y} / l ; r^{\prime}(x)=k b v^{\prime}(x)=k b(1-2 x / l) ; x$ is a coordinate calculated from the point of application of the force $P$. The balance equation for a work of the internal and external forces is written in the form:

From here, taking into account:

$$
M_{y} 2 \theta=P_{y} \delta-r_{0} \frac{l \delta}{2}+\Delta P \frac{\delta}{2}-2 \int_{0}^{l / 2} r^{\prime} v^{\prime} d x .
$$

$$
\frac{\Delta P \delta}{2}-2 \int_{\theta}^{l / 2} r^{\prime} v^{\prime} d x=\frac{\Delta P \delta}{2}-\frac{\delta^{2} k b l}{3}=0
$$

we get:

$$
P_{y}=\frac{8 M_{y}}{l}
$$

This solution, obtained by the kinematic method, is an approximation from above. Therefore, let us consider a solution by the static method, using a yield condition $M \leq M_{y}$ and the balance equations:

$$
P-\int_{0}^{l} r d x=0 ; \int_{0}^{l / 2} r x d x=M .
$$

Taking into account the uniform distribution of the foundation reaction up to an appearance of the plastic hinge, the same formula is obtained (1). 
Thus, the solutions by the both methods of the theory of limiting balance coincide, which means an accuracy of the obtained formula and a truth of the limiting value of the force for the adopted model of the system.

\subsection{A yield point at arbitrary arrangement of the concentrated force}

Let us now consider a beam loaded with a concentrated force applied at a certain distance $\eta l$ ( $0 \leq \eta \leq 0,5$ from the beginning of the beam (Fig. 2a). In this case, let us also assume that the bottom of the beam does not come off the foundation due to a constant load uniformly distributed along the entire length of the beam. In this case, the balance equations of the system can be written in the form:

$$
\begin{gathered}
P=\frac{\left(r_{1}+r_{2}\right) l}{2} \\
\left(\frac{1}{2}-\eta\right) r_{2}+\left(\frac{1}{6}-\frac{1}{2} \eta\right)\left(r_{1}-r_{2}\right)=0 \Rightarrow r_{2}=\frac{3 \eta-1}{2-3 \eta} r_{1} .
\end{gathered}
$$

The greatest bending moments can occur in the sections of our beam with the coordinates $x=\eta l, x=x_{0}$, which, with the yield conditions, can be written in the form:

$$
\begin{gathered}
M(\eta l)=\frac{l^{2}(1-\eta)^{2}}{6}\left[r_{1}(1-\eta)+r_{2}(2+\eta)\right] \leq M_{y} ; \\
M\left(x_{0}\right)=r_{1} \frac{x_{0}{ }^{2}}{2}-\left(r_{1}-r_{2}\right) \frac{x_{0}{ }^{3}}{6 l}-\left(r_{1}+r_{2}\right) \frac{l\left(x_{0}-\eta l\right)}{2} \geq-M_{y}^{-},
\end{gathered}
$$

where

$$
x_{0}=\frac{r_{1} l}{r_{1}-r_{2}}\left(1-\frac{r_{2}}{r_{1}}\right)
$$

is a coordinate of the largest negative moment.

Thus, the problem of finding $P_{y}$ (a yield point) for a given $\eta$ is reduced to finding the maximum largest value of the function (2) when the conditions (4) and (5) and the equation (3) are satisfied. For each $\eta(0-0.5)$ of the conditions (4) and (5) with the involvement of (3) from (2) the values $P$ are determined, the largest of which is a limiting value of the force $\mathrm{P}_{\mathrm{T}}$, corresponding to appearance of a plastic hinge under the concentrated force (a positive hinge with the moment $M_{y}$ ) or in the section to the right of the force (a negative hinge with the moment $-M_{y}^{-}$).

The Figure $2 \mathrm{~b}$ shows the dependence $P_{y}=P_{y} l / 8 M_{y}$ on the place of force application for three types of beams: $M_{y}^{-}=M_{y}, M_{\mathrm{T}}^{-}=0,5 M_{y}$ and $M_{y}^{-=0}$.

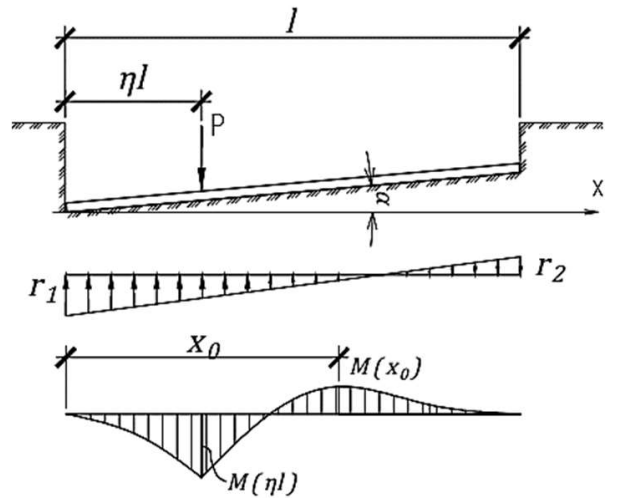

a)

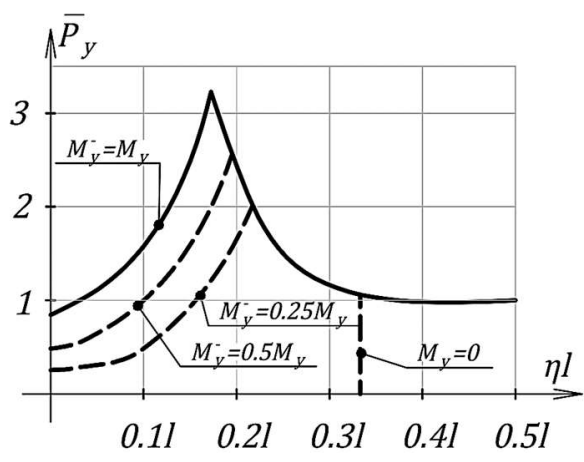

b)

Fig. 2. a) design diagram of a beam; b) dependencies of a limiting force $P_{y}^{\prime}$ on $\eta l$. 
As can be seen from the graphs, when the force is close to the edge, the yield point arises by forming a negative hinge with the moment $-M_{y}^{-}$. With distance from the edge, the values of the limiting force increase and reach the maximum value: $P_{y} \approx 3.18 M_{y} / l$ at $\eta \approx 0.17$ for $M_{y}^{-}=M_{y} ; P_{y} \approx 2.45 M_{y} \mathrm{l}$ at $\eta \approx 0,21$ for $M_{y}^{-}=0.5 M_{y}$ and $P_{y} \approx 1.27 M_{y} / l$ at $\eta=1 / 3$ for $M_{y}^{-}=0$. The plastic hinges then start being formed under the force $P_{y}$, which diminishes while approaching the middle of the beam. Moreover, the smallest value $P_{y}$ at $M_{y}^{-} \leq M_{y}$ occurs when the force is applied to the end of the beam $(\eta=0)$. For example, when $M_{y}^{-}=$ $M_{y}$ this force is 1.185 times less than $P_{y}$, applied to the middle $(\eta=0,5)$.

\subsection{Load corresponding to the limiting state}

After the formation of the first plastic hinge, two scenarios are possible: a further strain continues without increasing the force, i.e., the limiting state sets in, and the beam turns into a mechanism; the further growth of strains (an increase in the opening angle of the plastic hinge with a possible formation of other zones of plastic straining) providing an increase in the load to the limiting value.

For the latter option, until the limiting state starts, all intermediate states of the system are statically admissible and kinematically possible. At the same time, it should be borne in mind that the strains can be so great that it is necessary to take into account a geometric nonlinearity of the system.

Let us consider a beam, loaded with the force $P$, applied in the middle. After the formation of the plastic hinge under the force $P$ let the beam continues being strained with further opening of the hinge (increase in the bend angle $\alpha$ ) until other plastic zones appear, i.e., until the next plastic hinges appear. Let us define the force corresponding to a given state, which will be considered a limiting one and denoted as $\mathrm{P}_{n p}$.

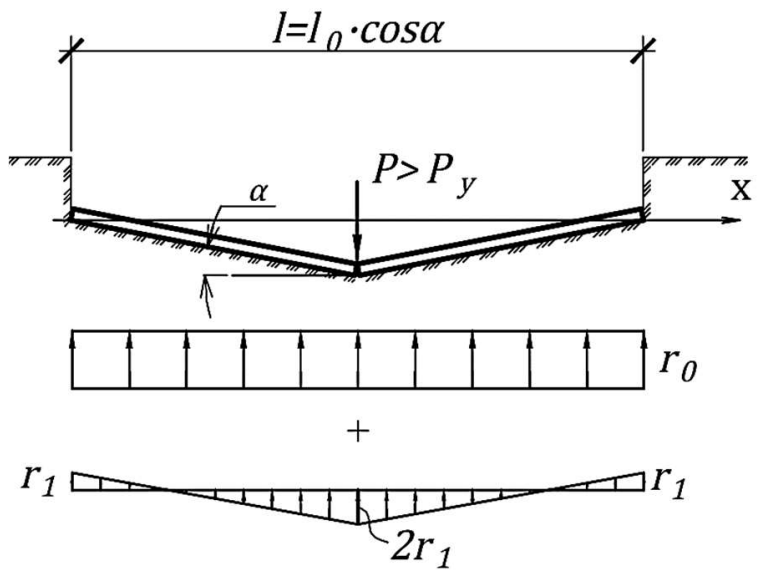

Fig. 3. Diagrams of strain of the beam after the formation of the plastic hinge.

Next, taking into account the symmetry of the scheme, let us consider the balance of a half of the beam (Fig. 4), where, instead of the initial length of the beam, its projection $l=l_{0} \cos \alpha$ is introduced, which, after the formation of the plastic hinge, changes as the beam are loaded further. The reaction of the foundation is divided into two parts so that relative to the point $O$ the moment from the uniformly distributed part is equal $M_{y}$, and the moment from the unevenly distributed part should be equal to zero. Then the balance equations can be written in the form:

$$
r_{0} \frac{l_{0}^{2} \cos ^{2} \alpha}{8}=M_{\mathrm{T}} ; \frac{P}{2}=r_{0} \frac{l_{0} \cos \alpha}{2}+r_{1} \frac{l_{0} \cos \alpha}{4} \text {. }
$$


Hence

taking into account

$$
P=\frac{8 M_{y}}{l_{0} \cos \alpha}+r_{1} \frac{l_{0} \cos \alpha}{2}
$$

$$
r_{0}-r_{1}=k b v_{1}, a r_{0}+2 r_{1}=k b v_{2}=k b
$$

$r_{1}$ is expressed, in terms of the opening angle of the plastic hinge:

$$
r_{1}=\frac{k b l_{0}}{6} \sin \alpha
$$

Then, substituting (7) into equation (6), we get:

$$
P=\frac{8 M_{y}}{l_{0} \cos \alpha}+\frac{k b l_{0}^{2}}{24} \sin 2 \alpha .
$$

Next, assuming that the bottom of the beam does not come off the foundation, the coordinate $x_{0}$ and the maximum negative moment, arising in the given section, are determined:

$$
x_{0}=\frac{-\left(r_{0}-r_{1}\right) l_{0} \cos \alpha}{3 r_{1}} ; M_{\max }^{-}=\left(r_{0}-r_{1}\right) \frac{x_{0}^{2}}{2}+r_{1} \frac{x_{0}^{3}}{l_{0} \cos \alpha} .
$$

Thus, the determination of the limiting load $P_{u}$, corresponding to the appearance of the second (negative) plastic hinge, is reduced to the problem of maximizing the function:

$$
P_{u}(\alpha)=\max \left(\frac{8 \mathrm{M}_{y}}{l_{0} \cos \alpha}+\frac{k b l_{0}^{2}}{24} \sin 2 \alpha\right)
$$

while fulfilling the restrictions:

$$
M_{\max }^{-} \geq-M_{y}^{-;} 0<x_{0}<l / 2 \text {. }
$$

It should be noted that, in the general case, to the specified constraints (9) one can add some constraints for the upsetting of the beam, as well as for the opening angle of the plastic hinge, since the plastic properties of the material have boundaries. The solution to the problem was implemented using the software package «Mathematica 9.0».

As a result of calculations, a dependence of the ultimate load $P_{u}$ on the parameter $\beta=$ $\left(k b /\left(4 M_{y} t\right)\right)^{-4}$, taking into account the «stiffness» of the foundation and the beam, is obtained, where $t=E I_{x} / M_{y}$ is a coefficient, connecting the stiffness of the beam with a yield moment of the section. Figure $4 \mathrm{~b}$ shows a graph of the dependence of these values, reduced to a dimensionless form: $\bar{P}_{u}=P_{u} l /\left(8 M_{y}\right), \beta l_{0} / 2$. In the same place, for comparison, the results of calculation $P_{u}$ by the finite element method for an elastoplastic model using the software package LIRA-SAPR are shown. To take into account the plastic properties of the elastoplastic model, after reaching the bending moment under the concentrated force of the value $M_{y}$, an ordinary hinge is inserted into the design scheme in this section with the addition of the moments $M_{y}$ on both sides.

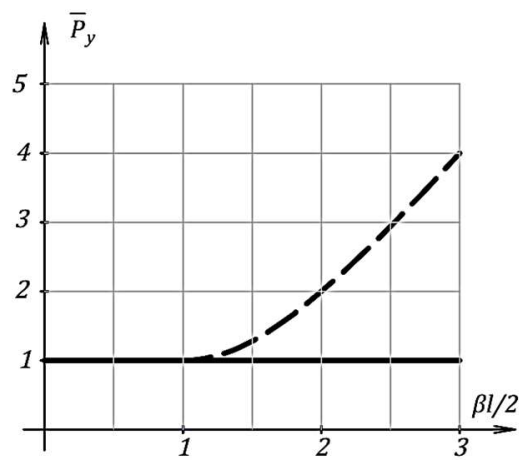

a)

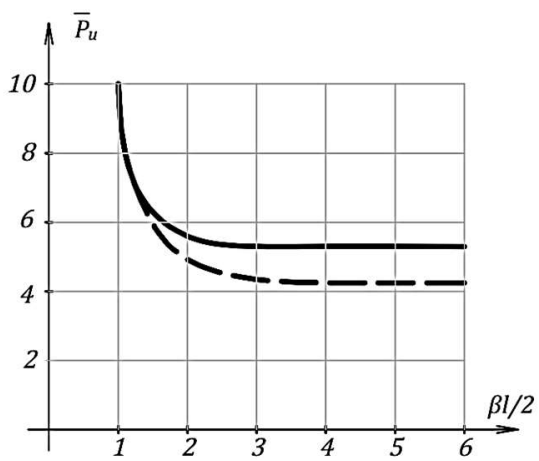

b)

Fig. 4. Dependences: a) of the yield limit $\bar{P}_{y} ;$ b) of the limiting load $\bar{P}_{u}$ on the parameter $\beta l_{0} / 2$ at $M_{y}^{-}=M_{y} .-$-is a rigid plastic model; - - is an elastoplastic model. 
Fig. 4a shows the results of calculations to determine a yield point, also reduced to a dimensionless form: $\bar{P}_{u}=P_{u} l /\left(8 M_{y}\right)$.

Fig. 5 shows the dependences of the ultimate load $\bar{P}_{u}$ и and the opening angle of the plastic hinge $\alpha$ on the parameter $\beta l_{0} / 2$ for a reinforced concrete beam with different lower and upper reinforcements: $M_{y}^{-}=0$ (without upper reinforcement); $M_{y}^{-}=0.5 M_{y}$ and $M_{y}^{-}=$ $M_{y}$ (equally reinforced).

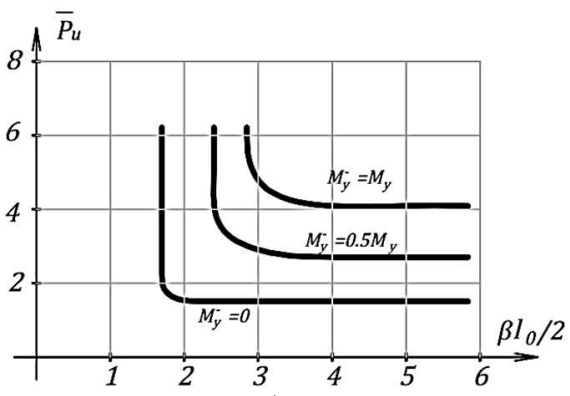

a)

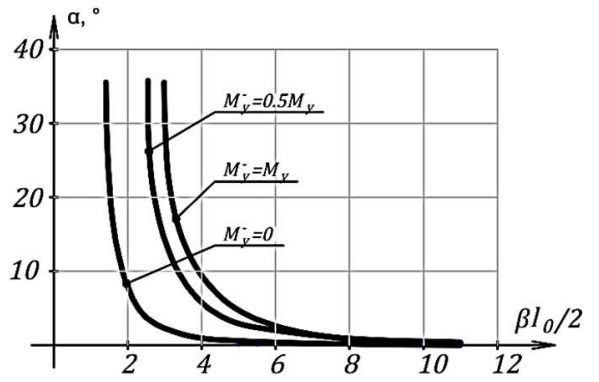

b)

Fig. 5. Dependences on $\beta l_{0} / 2$ : a) of the limiting load $\bar{P}_{u} ;$ b) of the opening angle of the hinge $\alpha$.

As can be seen from Fig. 4a, the graphs of the appearance of the first plastic hinge (under the force $P$ ) for the rigid-plastic and elasto-plastic models of the beam coincide only for the short rigid beams, the length of which $l_{0} \leq 2 / \beta$. As for the long $\left(l_{0}>2 / \beta\right)$ elastoplastic beams, a plastic hinge is formed at a higher force, which at $l_{0} \geq 4 / \beta$ can be accepted with the sufficient accuracy $P_{y}=4 \beta M_{y}$. The difference is explained by elastic wave-like damped deflections of the beam, preceding the appearance of the plastic hinge, which are neglected in the rigid-plastic model.

The second plastic hinges appear with negative opening. In this case, the values of the concentrated force $P_{u}$ for the adopted rigid-plastic model are greater than those for the elastoplastic model, which is also explained by the neglect of the elastic strains. Moreover, for all the beams $l_{0} \geq 5.4 / \beta$ long, the difference is constant and amounts to $40 \%$.

As can be seen from the graphs in Fig. $4 \mathrm{~b}$ and $5 \mathrm{a}$, the negative plastic hinges are not formed in all the beams, but only in the rather long ones. The short beams can be strained without the formation of the other plastic zones at any high load values. The limiting length of the beam and the corresponding $\bar{P}_{u}$ and $\alpha_{u}$, when the formation of plastic hinges becomes impossible, are:

$-l_{u}=2.82 / \beta, \alpha_{n p}=35.5^{\circ}$ и $\bar{P}_{u}=1.84$ for the beams with $M_{y}^{-}=0$;

$-l_{u}=5.10 / \beta \alpha_{n p}=35.5^{\circ}$ и $\bar{P}_{u}=4.85$ for the beams with $M_{y}^{-}=0.5 M_{y}$;

$-l_{u}=5.96 / \beta \alpha_{n p}=35.5^{\circ}$ и $\bar{P}_{u}=7.02$ for the beams with $M_{y}^{-}=M_{y}$.

With an increase in the length of the beam, $\bar{P}_{u}$ decreases and practically reaches the smallest values for the lengths of the beams indicated below:

$-l_{0} \geq 2.05 / \beta, \bar{P}_{u}=1.51$ for the beams with $M_{y}^{-}=0$;

$-l_{0} \geq 4.51 / \beta, \bar{P}_{u}=3.95$ for the beams with $M_{y}^{-}=0.5 M_{y}$;

$-l_{0} \geq 5.40 / \beta, \bar{P}_{u}=5.73$ for the beams with $M_{y}^{-}=M_{y}$.

It should be noted that for the long beams the second plastic hinge arises at a small opening angle of the average plastic hinge, but as the length of the beams approaches the above values $l_{u}$ (Fig. 5b) a sharp increase in the opening angle is observed, which reaches the same value for all cases -35.5 at $l_{0}=l_{u}$. 
Thus, a force $P_{\mathrm{T}}$ must be applied to form a plastic hinge in the middle of the beam. The further strain of the beam to the formation of the negative plastic hinges requires a significant increase in the load to a value, that can be taken as a limiting state of the beam, since the further strain of the beam (see Figure 6) is associated with small changes in the load under a significant increase in the opening angle of the middle hinge and an increase in the plastic zones, which, starting from those negative hinges appearing at $P=P_{u}$, approach the middle of the beam up to the distance $l_{y} / 2$. Further, with an infinite increase in the load, the beam is strained only due to the opening of the middle plastic hinge.

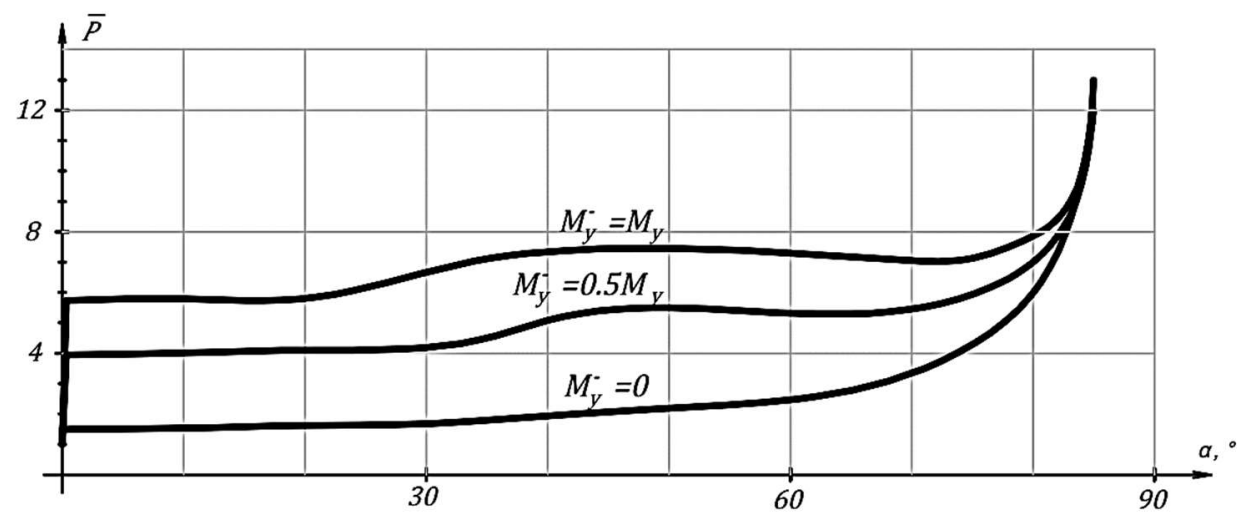

Fig. 6. A change of $\bar{P}_{u}$ upon opening of the plastic hinge for the beam $\beta l=10$.

\section{Conclusion}

1. Straining of the beam on an elastic foundation under the influence of the concentrated load has been studied using the methods of the theory of limiting balance.

2. The limiting values of the load have been derived analytically, and the forms of straining of the beam at the moment of the appearance of the first plastic strains and in the limiting state have been established.

3. The dependences of the indicated loads on the coefficient, generalizing the stiffness of the foundation and the beam, have been obtained, which are shown in the form of the graphs.

4. A comparative analysis of the results of the studies of the adopted model has been carried out with the numerical studies of the elastoplastic beam.

5. The analytical expressions and graphs, obtained in this work, can be useful in the practical calculations of beam structures on an elastic foundation in assessing the bearing capacity of beams, as well as in determining a yield point $P_{y}$ of short beams $l_{0} \leq 2 \beta$ long.

\section{References}

1. A.A. Gvozdev. Calculation of the bearing capacity of structures using the method of limiting balance. M.: Stroyizdat, 280 (1949).

2. A.P. Sinitsyn. Calculation of beams and slabs on an elastic foundation beyond the elastic limit. M.: Stroyizdat, 176 (1974).

3. V.G. Nizameev, E.R. Teregulova. Method for assessing the bearing capacity of reinforced concrete slabs lying on the foundation being strained, Izvestija KGASU 2 (12), 135-141 (2009).

4. I.G. Teregulov, R.A. Kayumov, V.G. Nizameev, E.R. Teregulova. Limiting state of slabs lying on the foundation being strained, News of TulSU, Natural Sciences 2, 108-111 (2008). 
5. V.G. Nizameev, F.F. Basharov. Investigation of the bearing capacity of a profiled flooring supported by a truss system, Science and business: ways of development 6 (84), 76-82 (2018).

6. V.G. Nizameev, F.F. Basharov, L.V. Nizameeva. Load carrying ability and optimal characteristics of the roof slab made of profiled sheeting, IOP Conf. Series: Materials Science and Engineering 890, (2020).

7. V.G. Nizameev, F.F. Basharov. Research on the bearing capacity of the corrugated steel decking under eccentric compression with supercritical operation, Izvestija KGASU $\mathbf{1}$, 95-101 (2016).

8. F.F. Basharov. The test truss plates with a span of $12 \mathrm{~m}$ of steel profiled decking brand H114-600 with the smallest wall thickness of 0,8 mm, Izvestija KGASU 4, 87-96 (2012).

9. V.G. Nizameev, F.F. Basharov. The method of optimization of design parameters of truss plates for profiled flooring, Science prospects 7 (106), 81-87 (2018).

10. A.R. Rzhanitsyn. Limiting balance of plates and shells. M.: Science, 288 (1983).

11. M.A. Vikulov, S.V. Efryushin. The method of constructing the region of the limiting bearing capacity of ideally plastic composite sections in a complex stress state, Scientific Bulletin of the Voronezh State University of Architecture and Civil Engineering. Construction and architecture 3 (31), 76-83 (2013).

12. V.I. Korobko, M.Yu. Prokurov, S.A. Morozov. Calculation of rectangular hingesupported plates, loaded with the arbitrarily applied concentrated force, by the method of limiting balance, Structural mechanics and design of structures 2, 2-8 (2011).

13. S.A. Morozov. Calculation of polygonal plates of constant thickness, loaded with a uniformly distributed load, by the method of limiting balance, Construction and reconstruction 1, 26-34 (2011).

14. Teodoru Iancu-Bogdan. Beams on Elastic Foundation. The Simplified Continuum Approach January, Bulletin of the Polytechnic Institute of Jassy, (LIX) 4, (2009).

15. I.B. Teodoru. Beams on elastic foundation - the simplified continuum approach, Buletinul Institutului Politehnic din Iasi, Tomul LV (LIX), Fasc. 4, (2009).

16. I.B. Teodoru: EBBEF2p - A Computer Code for Analysing Beams on Elastic Foundations, Intersections 6, (2009).

17. D. Dinev. Analytical Solution of Beam on Elastic Foundation by Singularity Functions, Engineering Mechanics 19 6, 381-392 (2012).

18. A. Beskopylny, E. Kadomtseva, G. Strelnikov. Static analysis of a reinforced bimodulus beam on elastic foundation, Theoretical Foundation of Civil Engineering, MATEC Web of Conferences 196, 02003 (2018). DOI: 10.1051/matecconf/201819602003.

19. R.A. Kayumov, B.F. Tazyukov, F.R. Shakirzyanov, I.Z. Mukhamedova. Large Deflections of Beams, Arches and Panels in an Elastic Medium with Regard to Deformation Shifts, Lobachevskii Journal of Mathematics 40 3, 321-327 (2019).

20. R. Khusnutdinov, Y. Konoplev, R. Kayumov. Features of stability loss of structures on an elastic foundation, IOP Conference Series: Materials Science and Engineering 890 (1), 012060 (2020). DOI: 10.1088/1757-899X/890/1/012060. 